\title{
About the nature of iron anodic activation in solutions of mineral acids
}

\section{Ya.G. Avdeev}

\author{
A.N. Frumkin Institute of Physical Chemistry and Electrochemistry, Russian Academy of \\ Sciences, Leninsky pr. 31, 119071 Moscow, Russian Federation \\ E-mail: avdeevavdeev@mail.ru
}

\begin{abstract}
Some aspects of the effect of hydrogen absorption on the anodic ionization of iron in solutions of mineral acids, both in the absence and in the presence of corrosion inhibitors, are discussed. It is shown that the process of iron anodic ionization in acid media is sensitive to the hydrogen content in the metal. The stronger the hydrogen content in the metal, the lower the rate of its ionization. In many cases, anodic activation of iron can result from the desorption of adsorbed hydrogen which, like an inhibitor, blocks the active centers of the metal ionization from the surface. The anodic activation of iron in inhibited acid solutions can be provoked by removal of adsorbed hydrogen from the metal surface, partial or complete desorption of the inhibitor, and formation of pits. It is noted that in inhibited acid solutions, even at potentials higher than the anodic activation potential, an adsorbed inhibitor can not only be partially retained on iron surface but also be adsorbed on a newly formed metal surface. The development of pitting upon anodic activation of iron results from partial desorption of an inhibitor from the metal surface. Pits form on inhibitor-free areas of the steel surface. The possibility of pitting corrosion of iron in inhibited solutions of mineral acids in the case of a shift of the corrosion potential by inhibitors to the pitting potential is discussed.
\end{abstract}

Keywords: anodic dissolution of iron, inhibition, activation potential, desorption potential, acid solutions.

Received: July 25, 2020. Published: October 26, 2020

doi: $\underline{10.17675 / 2305-6894-2020-9-4-10}$

This article was written in the wake of the review recently published in IJCSI [1] discussing the reasons for the anodic activation of steels in inhibited acid solutions. The author of the review comes to the conclusion that upon sufficiently strong anodic polarization of steels, their surface transforms to a "specific structurally disordered quasi-liquid state with shortrange order characteristic of a two-dimensional adsorption layer in an electrolyte." Under these conditions, desorption of any corrosion inhibitors, regardless of their chemical nature, will occur from the metal surface, which causes the anodic activation of steel. This approach can certainly be considered as a possible reason for the anodic activation of steels in inhibited acids, but we believe that the problem is much broader.

Anodic activation of iron and nickel is observed in acid solutions that contain no corrosion inhibitors. Slowly recorded potentiostatic curves of nickel anode in $\mathrm{H}_{2} \mathrm{SO}_{4}$ solution 
[2] and of iron electrode in $\mathrm{H}_{2} \mathrm{SO}_{4}$ and $\mathrm{HCl}$ solutions [3-5] have broken line shapes. The self-activation of nickel is attributed to the anodic initiation of submicroscopic steps and protrusions that serve as mobile centers where a metal reacts with solution components [2]. The self-activation of iron upon anodic polarization is largely the result of hydrogen absorption in contact with acid solutions [3-5]. Hydrogenated iron contains two forms of hydrogen. Absorbed hydrogen is incorporated into the metal bulk, whereas adsorbed hydrogen is present on its surface. Both forms exist in equilibrium with each other. The hydrogen atoms adsorbed on the surface of iron, similarly to an inhibitor, block the active centers of its ionization.

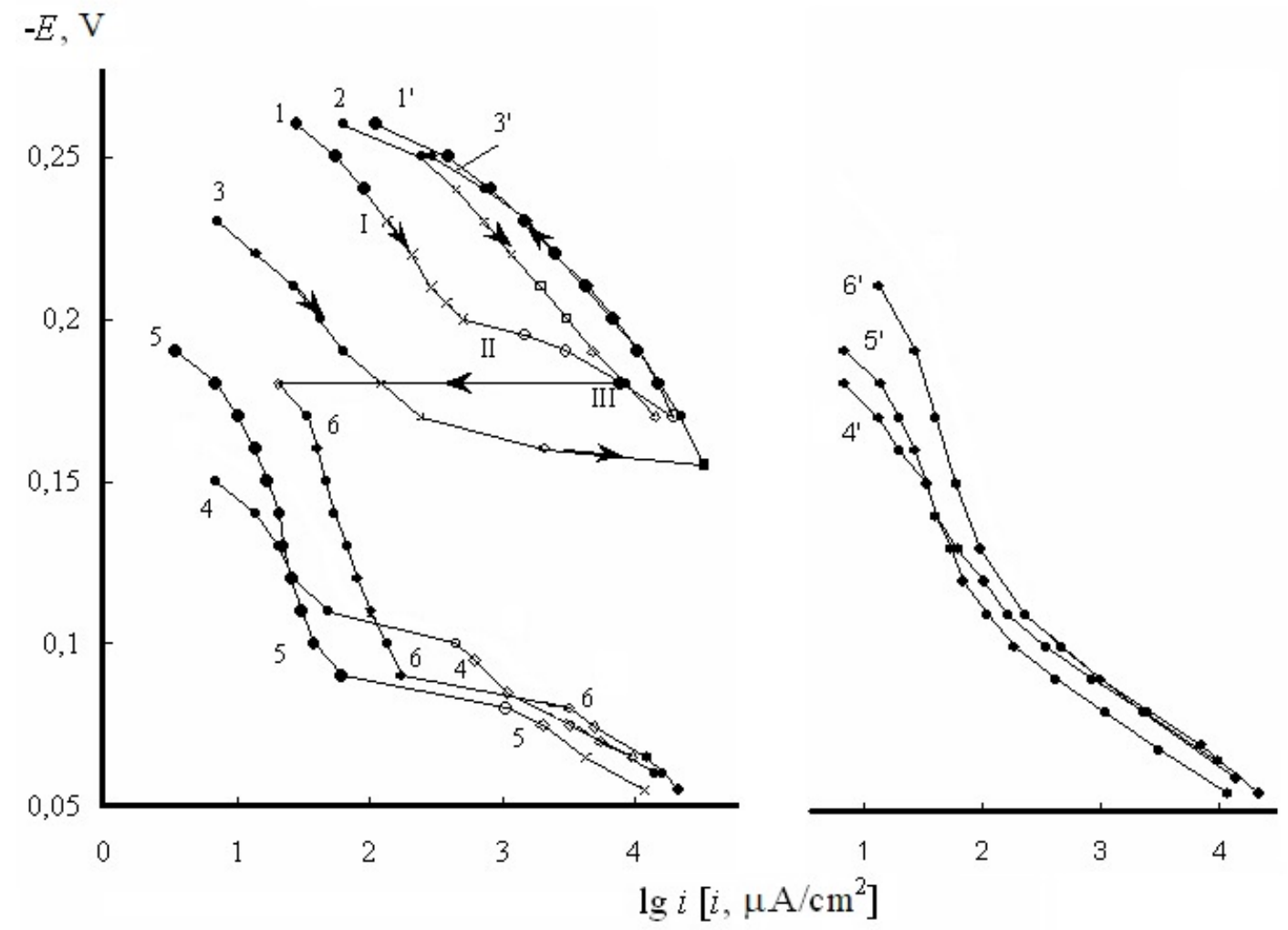

Figure 1. Curves of anodic polarization of iron in positive (without an accent) and negative direction (with an accent) in $1 \mathrm{M} \mathrm{HCl}$ without additives $\left(1,1^{\prime}, 2,3,3^{\prime}\right)$ and with addition of 30 mM PA $\left(4,4^{\prime}-6,6^{\prime}\right): 1,1^{\prime}, 2,4,4^{\prime}, 6,6^{\prime}$ - without preliminary hydrogen absorption by the electrode; 2 - repeated polarization increase; $3,3^{\prime}, 5,5^{\prime}$ - after hydrogen absorption; 6, $6^{\prime}$ PA was added after electrode activation at $E=-0.18 \mathrm{~V}$. Time of electrode exposure at the set potential (min.): • $-3 ; \times-5 ; \diamond-10 ; \bullet-20 ; \mathrm{o}-40$ [5].

A clear illustration of this statement was obtained in a study on the effect of hydrogen absorption on the anodic dissolution of iron in $1 \mathrm{M} \mathrm{HCl} \mathrm{[5]} \mathrm{(Figure} \mathrm{1).} \mathrm{The} \mathrm{anodic} \mathrm{curve} \mathrm{of}$ iron (curve 1) pre-exposed at the corrosion potential $\left(E_{\text {cor }}\right)$ in $1 \mathrm{M} \mathrm{HCl}$ comprises three sections. Section I corresponds to the process that occurs on the metal "poisoned" with atomic hydrogen. Section II corresponds to the anodic activation of iron due to the removal of atomic hydrogen from the surface. In section III, the anodic ionization of iron occurs on 
the metal free of adsorbed hydrogen. The reverse sweep of the anodic curve (curve 1') also characterizes the ionization of iron on the metal free of hydrogen. If the potential $(E)$ of the iron electrode is increased immediately after recording curve 1', then anodic curve 2, like curve 1', will have one linear section, but the recorded anodic currents will be slightly smaller due to the slight hydrogen absorption on the metal at $E$ close to $E_{\text {cor. }}$. Anodic curve 3 of iron that was pre-hydrogenated cathodically also contains sections I-III, but, unlike curve 1 , section I is characterized by smaller currents and is more extensive due to a higher content of hydrogen in the metal. Thus, the anodic activation of iron in acid solutions results from the removal of adsorbed hydrogen, which inhibits the anodic ionization of iron, from the metal surface.

The processes occurring during the anodic ionization of iron in inhibited acid solutions are more complex. Let us consider them in the case of iron anodic ionization in $1 \mathrm{M} \mathrm{HCl}$ containing propargyl alcohol (PA). PA adsorbed on iron surface at $E_{\text {cor }}$ significantly inhibits the anodic reaction (curve 4$)$, but as the activation potential $\left(E_{\text {act }}\right)$ is reached, like in the background medium, a sharp current increase corresponding to the anode nonpolarizability region occurs. After the non-polarizability region, there is a third region of the anodic curve with a smaller Tafel slope than in the first region. Forced cathodic hydrogenation of iron (curve 5) shifts $E_{\text {act }}$ to a more positive region. In contrast, the adsorption of PA on nonhydrogenated metal (curve 6) weakens the inhibition of the anodic reaction by the inhibitor. In media inhibited with PA, like in the background, a hysteresis of the forward and reverse anodic curve scans is observed. One can see that hydrogen absorption by iron in an inhibited medium affects its anodic activation, but anodic activation of the metal occurs even in the case of minor hydrogen absorption by the electrode (curve 6). A natural conclusion is that the anodic activation of iron in inhibited acid results from the desorption of hydrogen and the inhibitor, or only the inhibitor, from the metal surface, but this is true to some extent only. In the course of iron anodic activation, the inhibitor is removed only from certain areas. Pits develop in these places [5]. It is the growth of pits that is largely responsible for the electrode activation. Such pits are visually observable on iron surface. It is interesting that PA can heal pits formed on iron in $1 \mathrm{M} \mathrm{HCl} \mathrm{upon} \mathrm{anodic} \mathrm{activation.} \mathrm{The} \mathrm{pattern} \mathrm{of} \mathrm{variation}$ of the anodic current versus time at $E=E_{\text {act }}+10 \mathrm{mV}$ shows that it increases at first, then the growth slows down, the current passes through a maximum and starts to decrease. The time dependence of the degree of iron protection by PA has the form shown in Figure 2. This is possible if pitting goes through the stages of growth and healing by an inhibitor [6]. Thus, at $E>E_{\text {act }}$, not only can the adsorbed inhibitor be retained on the steel surface, but its additional adsorption on the newly formed inner pitting surface can also take place.

In solutions of mineral acids, the anodic activation of iron results from different rates of anodic ionization of hydrogenated and non-hydrogenated iron. Hydrogenated iron dissolves rather slowly. When $E_{\text {act }}$ is exceeded, the metal surface is freed from adsorbed hydrogen and anodic ionization of iron occurs at higher rates typical of the process on nonhydrogenated metal. 


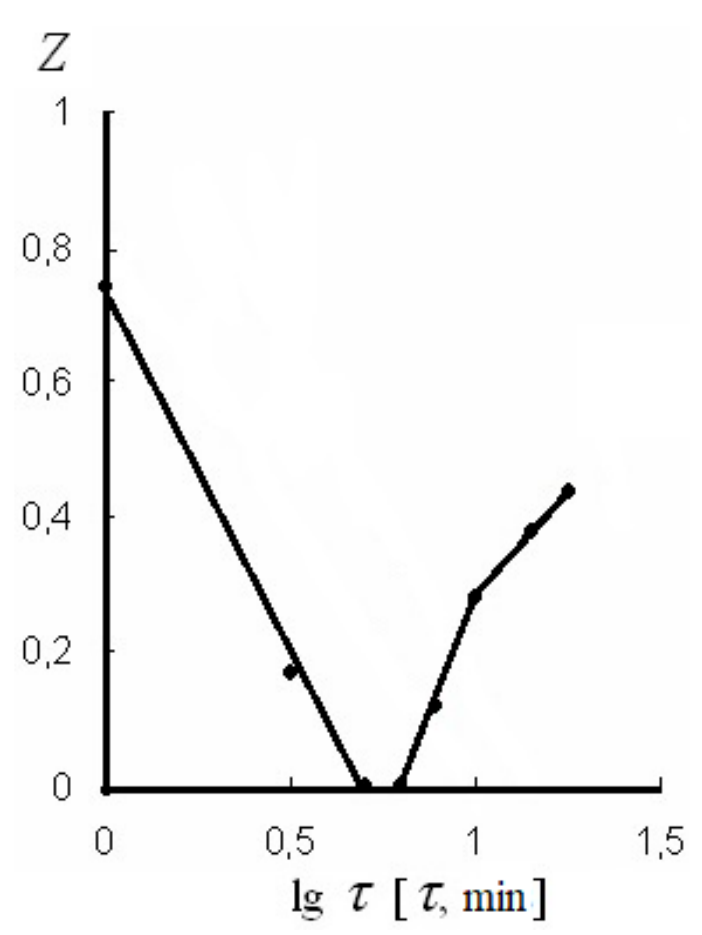

Figure 2. Degree of iron protection $v s$. $\lg \tau$ during PA desorption; $E=E_{\text {act }}+10 \mathrm{mV}$. PA concentration: $10 \mathrm{mM}[6]$.

The nature of iron activation in inhibited acid media is more ambiguous. It is important that this process is sensitive to the hydrogen content in iron [3-5]. The anodic activation of iron in inhibited acid solutions can be stimulated by removal of adsorbed hydrogen from the metal surface, partial or complete desorption of the inhibitor, and formation of pits. It is not always possible to distinguish the contribution of each of these factors to iron activation. Moreover, the question of how the anodic polarization of a metal causes the removal of hydrogen and inhibitors from its surface remains open.

In the above example, both in the background and in the inhibited acid, the $E_{\text {act }}$ of iron is considerably more positive than its $E_{\text {cor }}$, in good agreement with the data discussed in the review [1]. However, cases where $E_{\text {cor }} \approx E_{\text {act }}$ on iron in inhibited media were also reported [7-9]. This is possible if the inhibitor added to the corrosive environment stimulates the cathodic process but efficiently inhibits the anodic reaction of iron. Such an inhibitor shifts $E_{\text {cor }}$ to $E_{\text {act }}$ For example, this situation is observed for iron in $1 \mathrm{M} \mathrm{H}_{2} \mathrm{SO}_{4}$ inhibited by PA (Figure 3) [7]. When a metal is operated in such an inhibited environment, this is extremely dangerous since there is a possibility that pitting corrosion would develop at $E_{\text {cor. }}$ Indeed, prolonged exposure of low-carbon steel samples in $\mathrm{H}_{2} \mathrm{SO}_{4}$ solutions inhibited by PA gives rise to shallow pits on the metal surface. It is extremely important to take this possibility into account in the industrial application of corrosion inhibitors [9]. 


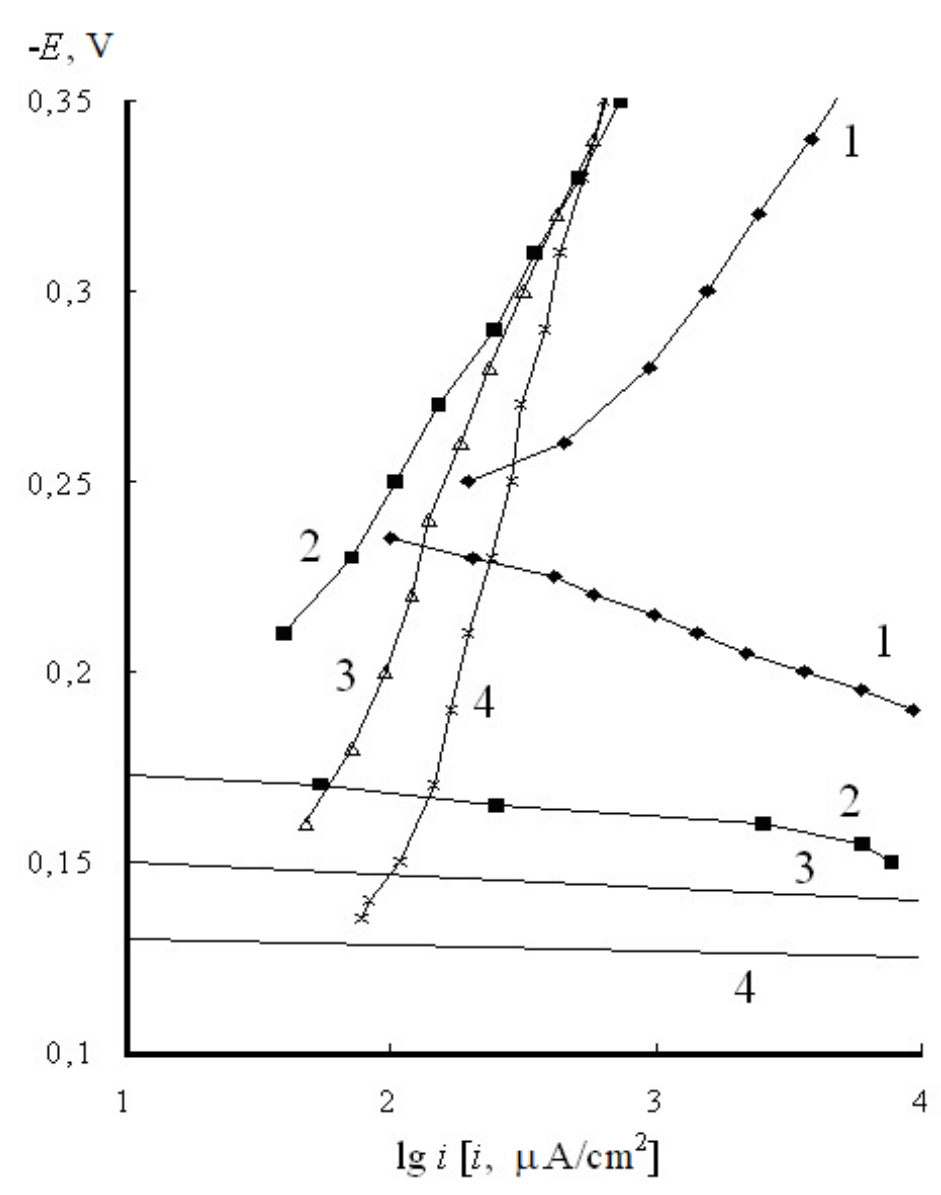

Figure 3. Polarization curves on iron in $1 \mathrm{M} \mathrm{H}_{2} \mathrm{SO}_{4}(1)$ in the presence of PA (mM): $2-1,3-$ $5,4-30[7]$.

\section{Conclusions}

The anodic activation of iron and steels is characteristic exclusively of background or inhibited acid solutions. In these environments, the hydrogen uptake by metals and especially by their surfaces occurs rather quickly. This indirectly indicates that there is a relationship between the metal anodic activation and hydrogen desorption processes. Removal of adsorbed hydrogen from the metal surface, partial or complete desorption of the inhibitor, and pitting should be considered as the reasons for the anodic activation of iron and steels in solutions of mineral acids. However, more specific details of each of the possible pathways of iron anodic activation require more thorough experimental and theoretical studies.

\section{Acknowledgements}

This study was carried out within the framework of the Program for Basic Scientific Research of the State Academies of Sciences for 2013-2020. The topic "Development of the fundamental scientific basis of the protective effect of inhibitors of metal corrosion in gas and condensed media, nanocomposites, paintwork and conversion coatings" (state registration number AAAA-A18-118121090043-0). 


\section{References}

1. M.A. Pletnev, Effect of inhibitors on the desorption potentials in the anodic dissolution of iron in acid solutions - A review, Int. J. Corros. Scale Inhib., 2020, 9, no. 3, 842-866. doi: $10.17675 / 2305-6894-2020-9-3-4$

2. V.M. Novakovskiy, G.N. Trusov and M.F. Fandeyeva, Ob osobennostyakh i prirode povedeniya nikelya do nachala vtoroi passivatsii (On the features and nature of the behavior of nickel before the beginning second passivation), Zashch. Met. (Protection of Metals), 1969, 5, no. 5, 503-510 (in Russian).

3. N.I. Podobaev and G.G. Klimov, Vliyanie navodorozhivaniya na rastvorenie zheleza i ingibirovanie $\mathrm{v}$ kislykh sul'fatnykh rastvorakh (The effect of hydrogenation on the dissolution of iron and inhibition in acidic sulfate solutions), Zashch. Met. (Protection of Metals), 1980, 16, no. 5, 611-615 (in Russian).

4. N.I. Podobaev and V.M. Larionova, Vliyanie vodoroda na ionizatsiyu zheleza i na razryad vodorodnykh ionov $\mathrm{v}$ ingibirovannom sul'fatnom rastvore (The effect of hydrogen on the ionization of iron and on the discharge of hydrogen ions in an inhibited sulfate solution), Zashch. Met. (Protection of Metals), 1995, 31, no. 3, 292-294 (in Russian).

5. N.I. Podobaev and Ya.G. Avdeev, The Effect of Hydrogenation on the Anodic Dissolution of Iron and Its Inhibition by Propinol in Hydrochloric Acid, Prot. Met., 1999, 35, no. 5, 484-488.

6. N.I. Podobaev and Ya.G. Avdeev, Joint Effect of Hydrogen and Propargyl Alcohol on the Kinetics of Electrode Reactions and Iron Corrosion Rate in Hydrochloric Acid, Prot. Met., 2000, 36, no. 2, 180-184. doi: 10.1007/BF02758343

7. N.I. Podobaev and Ya.G. Avdeev, Specific Effects of Propargyl Alcohol and Propargyl Chloride on an Iron Electrode in Inorganic Acids, Prot. Met., 2000, 36, no. 3, 251-257. doi: $10.1007 / \mathrm{BF} 02758401$

8. N.I. Podobaev and Ya.G. Avdeev, Temperature and Time Effects on the Acid Corrosion of Steel in the Presence of Acetylenic Inhibitors, Prot. Met., 2001, 37, no. 6, 529-533. doi: 10.1023/A:1012855211234

9. N.I. Podobaev and Ya.G. Avdeev, The Effect of Several Anions and Inhibitors Mixed with Propargyl Alcohol on Electrode Reactions and Corrosion of Steel in Sulfuric Acid, Prot. Met., 2001, 37, no. 1, 13-17. doi: 10.1023/A:1004873231812 